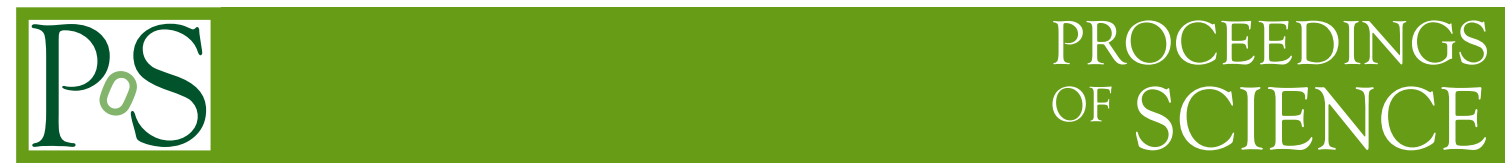

\title{
Neutrino Astronomy in the IceCube Era
}

\author{
Todor Stanev* \\ Bartol Research Institute and Department of Physics and Astronomy, University of Delaware, \\ Newark, DE 19716, U.S.A. \\ E-mail: stanevabartol.udel.edu
}

\begin{abstract}
We present the results from four years detection of the IceCube neutrino observatory of high energy astrophysical neutrinos. The search for such neutrinos and their sources is an extremely important part for the search of very powerful astrophysical objects where cosmic rays are accelerated to very high energy. We compare the directions from which these high energy neutrino arrive with the directions of gamma-ray and hard X-ray sources.
\end{abstract}

Frontier Research in Astrophysics - II

23-28 May 2016

Mondello (Palermo), Italy

${ }^{*}$ Speaker. 


\section{Introduction}

When I was asked to give a talk with this title I had a problem with the word astronomy. On the other hand, when the IceCube experiment detected very high energy neutrino events. the majority of which are astrophysical, we started using the term neutrino astronomy. And scientists all over the world started searching for the sources of these astrophysical neutrinos. For readers that are not familiar with neutrinos, we should say that neutrinos can travel in the Universe even longer than optical light without being absorbed. There was recently a private discussion of what is the redshift from which $10^{15} \mathrm{eV}$ neutrinos can arrive to us without interacting, is it $z$ of 9 or 12 ?

Astrophysical neutrinos are produced by high energy cosmic rays. They may be the result of hadronic interactions of the accelerated cosmic rays in the vicinity of the sources if the matter density of their sites is significant. They could also be produced inside the accelerating astrophysical object in photoproduction interactions $p+\gamma \rightarrow p+\pi^{ \pm}+\ldots$ with the local photon field. A typical neutrino production model of this type is the one by Eli Waxman \& John Bahcal [1] that attempted to set an upper limit on the fluxes of astrophysical neutrinos based on the emissivity of UHECR in the Universe and on their acceleration spectrum. The source of UHECR and astrophysical neutrinos in this model are the gamma ray bursts (GRB). Very high energy neutrinos have to be produced in photoproduction interactions of the highest energy cosmic rays in their propagation from the acceleration sites to us.

Then there is the question: What is the IceCube Era and when did it start? Is it when the experiment was completed in December 2010, or when the first detection of astrophysical neutrinos was announced. Even before that: What is IceCube and how does it work.

We will try to answer all these questions before we turn to the neutrino astronomy part. How should we look for the sources of the astrophysical neutrinos, and what are the main problems in doing neutrino astronomy.

\section{The IceCube Experiment}

The structure and the size of the IceCube experiment is shown on the lefthand side of Fig 1. IceCube is $1 \mathrm{~km}^{3}$ detector constructed beetween 1.5 and $2.5 \mathrm{~km}$ below the surface of the ice at the South Pole. It consists of 80 strings that contain 60 photomultipliers (PMT) each that look down. The average distance between the strings is 125 meters. There are also 8 different strings that are shorter and are positioned at the bottom where the ice is very clear. On the top of the ice is an extensive shower detector which is called IceTop.

Each string was built by first drilling a hole in the ice and then pouring hot water in that hole. Hot water was pumped until it reached the desired depth. During that time, while the water in the hole was not frozen, the string with the PMTs was lowered in the hole and hold in the right position until the water froze. Initially this process took a lot of time. The collaboration became much better during the construction and during the last years of the deployment more than 10 strings were deployed each year. The construction was finished in December 2010.

When a charged particle penetrates through the ice it emits Cherenkov light. The IceCube PMTs detect the light and estimate the total pathlength of charged particles above the Cherenkov threshold. When, for example, a high energy muon travels through IceCube it interacts with the ice 
and thus produces many charged particles. By counting their energy loss IceCube calculates what is the deposited energy by the muon (or group of muons) in the detector. After that we can estimate what the energy of the muon was before it entered the detector and on the serface. Detecting such events is one of the two event types that IceCube sees - we will call them muon tracks. The other type of events is when a neutrino interacts with the ice inside the detector. These are the neutrino cascades.

These two types of events have different qualities. The arrival direction of the muon tracks is well measured, with an error less than $1.2^{\circ}$. One the other hand the energy of the muon is estimated from the energy loss of the muon inside the detector. Since the energy loss is a stochastic process it could be wrong by a large factor. In the case of neutrino cascades, when the neutrino interacts inside IceCube, the energy of the event is measured much better since the detector can see how many charged particles the interaction produced. The average error in the energy is of order $10 \%$ to $15 \%$. The arrival direction of the neutrino is, however, much more difficult to determine as the event looks like an explosion inside the detector. The error bars of the direction of these events are of order of $10^{\circ}$.

The muon tracks are only produced by muon neutrinos and antineutrinos. Those of them that go upwards inside the detector, after passing through the Earth, are obviously due to neutrinos. Muon tracks that enter Icecube from above could be muon neutrinos or muons generated in atmosphere by interacting cosmic rays. These atmospheric muons and neutrinos have a well known energy spectrum that extends to about $1000 \mathrm{TeV}$. The measurements by IceCube of the atmospheric muon and electron neutrinos [2,3] confirmed the theoretical predictions for such events, as e.g. [4].

The origin of the cascade events is more complicated. They could be generated by charged current (CC) interactions of electron and tau neutrinos. When tau neutrinos interact they produce a double bang [5], two centers of particle production - the first from the interaction and the second one from the produced tau lepton decay. At energy of $10^{6} \mathrm{GeV}$ the tau decay length is about 50 meters, not much bigger than the vertical distance between two PMTs (17 meters). It is not obvious that IceCube can distinguish betwee electron and tau neutrino interactions. Cascade events will also be produced by neutral current $(\mathrm{NC})$ interactions, where only a fraction of the neutrino eneregy is released and the rest is carried out by the secondary neutrino. The interaction cross section for $\mathrm{NC}$ collisions is less than $1 / 2$ of that for charged current, but all three types of neutrinos can generate $\mathrm{NC}$ cascade events. Obviously in the case of $\mathrm{NC}$ events the neutrino energy would be underestimated. A good description of the IceCube experiment was published after the experiment was deployed [7].

\subsection{The IceCube Era}

In my mind the IceCube era started three years ago, when the results of the first three years of the experiment were published [8,9]. These publications described 37 neutrino events, mostly neutrino induced cascades, with energy exceeding $10^{4} \mathrm{GeV}$, i.e. $10 \mathrm{TeV}$. The fact that the number of neutrino induced cascades was more than three times bigger than the number of muon tracks was initially surprising for the collaboration. The main idea before and during the construction of the experiment has been to see first upward going muon tracks. For this reason the PMTs were pointing downwards. 
It became soon obvious for the collaboration that we indeed expect more cascades than muon tracks if the detected astrophysical neutrinos contain equal number of electron, muon, and tau neutrinos because of the neutrino oscillations on the long way from the sources to us. Then we will have two neutrino types generating cascades plus three neutrino types generating cascades in $\mathrm{NC}$ interactions. If we take (for simplicity) the $\mathrm{NC}$ cross section to be one half of the $\mathrm{CC}$ cross section we will obtain a ratio of cascades to tracks to be 3.5 , although in NC collisions only about $1 / 4$ of the neutrino energy is deposited in the detector.

These results were obtained using a more complicated geometry of the experiment that guarantees that events started inside the detector. Such events are called High Energy Starting Events (HESE) and the requirements for their detection are shown in the righthand panel of Fig. 1. PMTs on top and on the sides of IceCube were used as a veto, i.e. there should not be a signal in these photomultipliers. Events should thus start in the middle of the detector, except in a small region where the ice is not transparent enough, probably because of very high volcanic activity at the time. Events coming up through the bottom of IceCube were not vetoed.

At the low energy end, just above $10 \mathrm{TeV}$, some of the events could be atmospheric neutrinos. For this reason the energy spectra of the astrophysical neutrinos were different when estimated above different threshold energy. There are always a bit softer than the classical $\mathrm{E}^{-2}$ acceleration spectra.
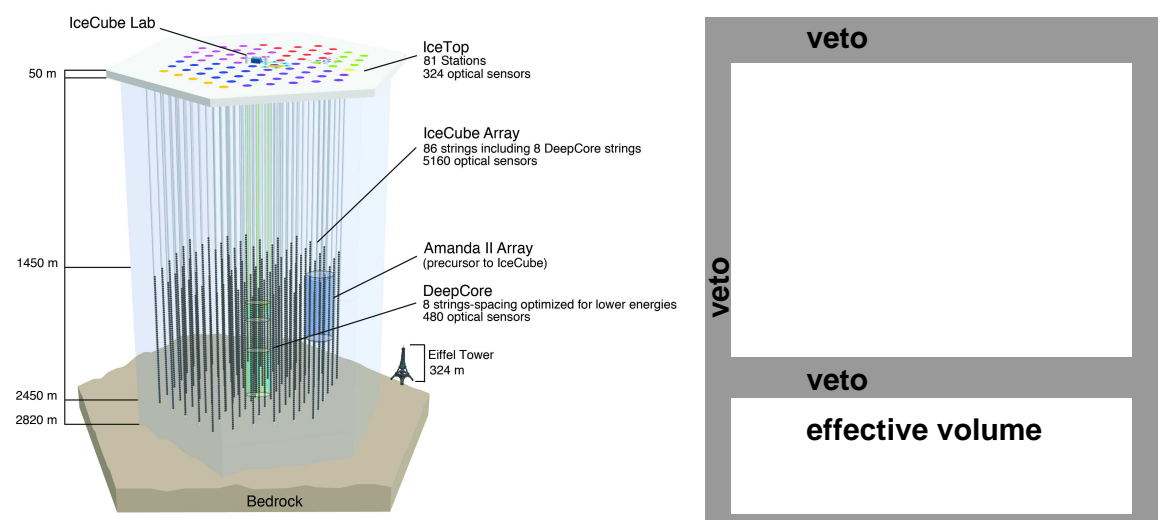

Figure 1: Lefthand panel: The IceCube detector; Righthand panel: Geometry of the IceCube high energy detection scheme of starting events.

\section{IceCube detection of astrophysical neutrinos}

It is obvious that the search for astrophysical neutrinos should start at high energy because the atmospheric neutrino background is too high at lower energy. In the case of IceCube the discovery started in 2012, when the Japanese group of IceCube members started looking for the highest energy events.

This analysis revealed 28 events that deposited in the detector between 30 and 1,200 $\mathrm{TeV}$ of energy [9] versus a background of $10.6_{-3.6}^{+5.0}$ events coming from atmospheric muons and neutrinos. The events included track events consistent with muon neutrinos and cascade events similar in 
shape with the previously discovered PeV events. Four of the track events start near to the top of the detector and point down, and are thus consistent with the atmospheric muons background of $6 \pm 3.4$ events. One of these events had hits in the IceTop surface air shower array IceTop, compatible in arrival time and direction. The two $\mathrm{PeV}$ events were also identified in this analysis.

After that IceCube released the data from the same analysis of one more year of observations [10], which revealed nine more high energy events. Most of these events were penetrating IceCube from above rather than from below. It was shown in the paper [9] that the effective area $A_{\text {eff }}$ of IceCube for downgoing neutrinos is higher than for upgoing events. The reason is that high energy neutrinos going through the Earth are absorbed. The absorbtion depends on the pathlength of the neutrinos inside the Earth. The absorbtion is highest (and the absorption energy threshold is lowest) for vertically upwardgoing events. Having these two facts in mind both initial surprises are easily explained. The last portion of the astrophysical neutrino detected by IceCube was published in the proceedings of the International Conference of Cosmic Rays in 2015 in The Hague [11]. The zenith angle of all these 54 events ( 39 cascades and 15 muon tracks) versus their energy is shown in the lefthand panel of Fig. 2. The same presentation includes also the newest results on atmospheric neutrinos and other types of astrophysical meutrino measurements.
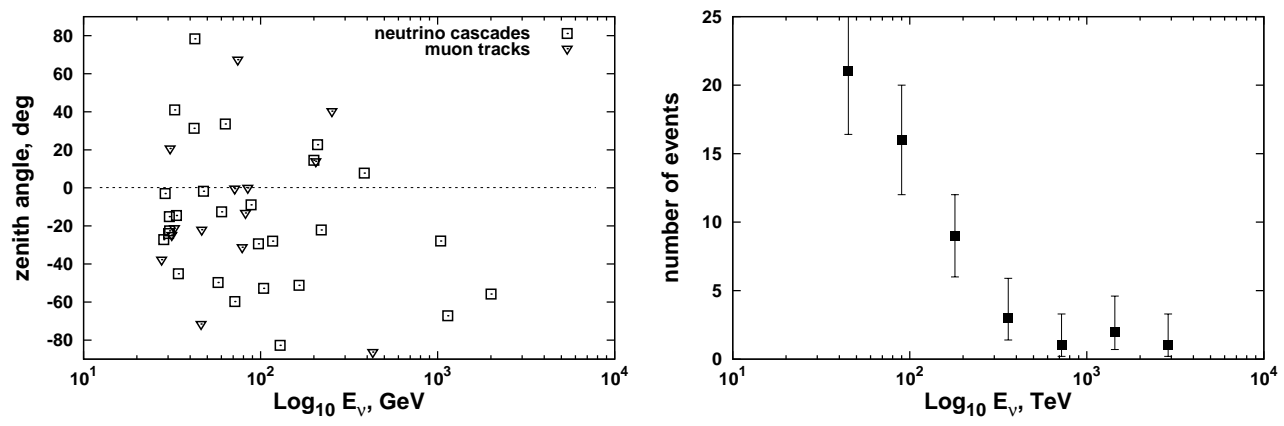

Figure 2: Lefthand pahel: Declination versus deposited energy in the detector for all high energy neutrinos in IceCube. Righthand panel: Energy spectrum of these 54 events.

The righthand panel shows the energy spectrum of these events. This is not an official IceCube results - the shown energy spectrum was made by me using the energy assigned by the collaboration to this fifty four events. Two events with energy slightly lower than $30 \mathrm{TeV}$ are included in the first group. The correct energy spectrum of the astrophysical neutrinos has to take account for the increasig neutrino interaction cross section. The official flux of astrophysical neutrinos detected in four years of IceCube observation is given in Ref. [11] normalized to energy of $100 \mathrm{TeV}$ in the form

$$
E^{2} \phi(E)=(2.2 \pm 0.7) \times 10^{-8}(E / 100 T e V)^{-0.58} \mathrm{GeV} \cdot \mathrm{cm}^{-2} \cdot \mathrm{s}^{-1} \cdot \mathrm{sr}^{-1}
$$

\section{Neutrino astronomy}

Although the available statistics of neutrino events that deposited more than $30 \mathrm{TeV}$ in IceCube is still very small, it is extremely interesting to compare the arrival directions of these neutrinos to other relevant signals. This would be the way to introduce neutrino astronomy. There is, however, 
a big problem there. As we already said 39 of the 54 high energy events are neutrino generated cascades, and the error bars on the arrival directions of these events is of order of $10^{\circ}$. If one adds to this error the huge distance from which these neutrinos might come, one can imagine the huge number of possible sources inside the error bars.

Fig. 3 shows the error bars of the thirty nine neutrino cascade events detected by IceCube. The muon track events with error bars less than $1.2^{\circ}$ are indicated with full squares (which on the scale of the cascades error bars are higher than the errors of the muon tracks). The event numbers of all IceCube events are attached to all events, cascades or muon tracks.

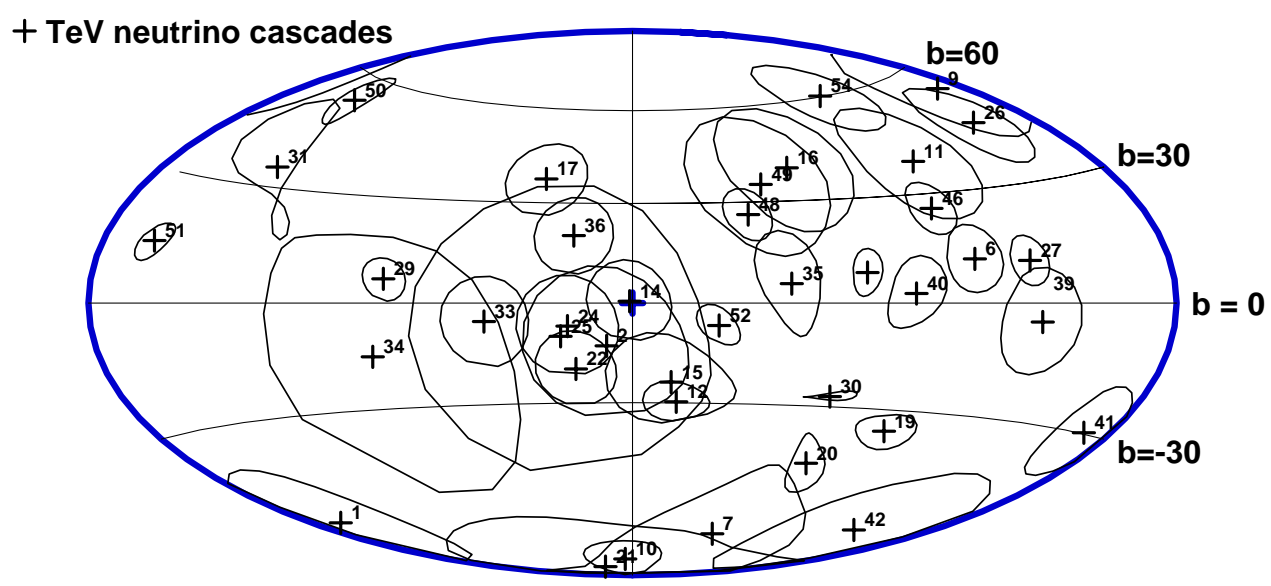

Figure 3: Arrival directions of the cascade signals in galactic coordinates. The error bars of the three PeV neutrino induced cascades are plotted with wider, better visible, line.

When we first look at Fig. 3 we might think that there is anisotropy in these events. Look, for example, at the region below and east of the Galactic Center where we have six intersecting error bars. The same is true also in the region of $b=30^{\circ}, l=300^{\circ}$ where we have five intersecting ones. Both these groups of events include one of the $\mathrm{PeV}$ events. IceCube has looked seriously at the possibility of anisotropy in this map, accounting for the size of the direction error bars, and has determined that there is no significant anisotropy there, inspite of the first impression.

The only thing we can do now, in the absence of anisotropy, is to plot the sources of other relevant signals together with the neutrino directions and have a better look at these coincident sources. An example of that is shown in Fig. 4 where we compare the Swift/BAT sources [12] directions to these of the IceCube events. There is obviously a concentration of the hard X-ray sources around the Galactic plane, and especially around the Galactic Center. There are also more sources in other areas of the map, away from the Galactic plane. One assumes that these are extragalactic sources and some of them could also be sources of ultrahigh energy neutrinos.

We show this graph not in order to analyze the concidental events, we only want to show how many concidences exist even in a moderately large catalog. Identifying possible sources should start with discovering similar known radiation sources within the error bars of all IceCube events. Doing this should start with a good discussion of what type of sources can produce both the signals in a catalog and high energy neutrinos. 


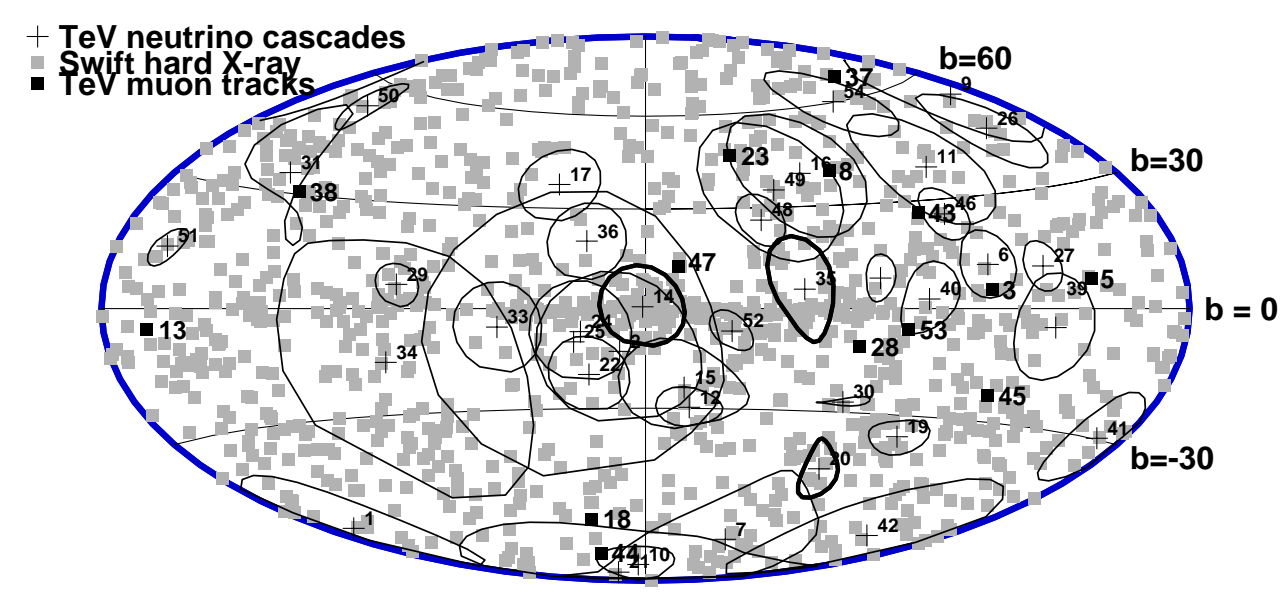

Figure 4: Arrival directions of IceCube signals to those of the Swift/BAT source catalogue.

As an example we could start the discussion with the sources of TeV $\gamma$-rays. Such sources do require acceleration of cosmic rays to moderately high energies. Many of these sources are identified in the TeVCat catalog [6]. Some of the $\gamma$-ray sources, however, are not suitable for sources of the $\mathrm{PeV}$ neutrinos, since they do not always require hadronic interactions. Gamma rays also interact with the CMB and EBL photons and produce electron/positron pairs. This way the gamma rays cascade in propagation to us.

Generally we are looking for sources that can accelerate cosmic ray nucleons up to more than $10 \mathrm{PeV}$ and have enough cosmic ray interaction targets around them. This could be similar to the $\mathrm{TeVCat}$ sources of $\mathrm{TeV} \gamma$-rays that are supernova remnants inside molecular clouds. They could also be surrounded by sources of energetic gamma rays, with which the high energy cosmic rays can also interact. These should be the main features of the high energy neutrino sources.

\section{Discussion and the Future}

Currently IceCube is much bigger than the existing observatories in the sea, such as Antares, so that we cannot expect soon a significant increase of statistics. When KM3NeT is completed it will be $\mathrm{km}^{3}$ detector, the same size a IceCube and will contribute to the detection of astrophysical neutrinos. There are several groups in the IceCube Collaboration that are pursuing different analysis methods from the one described above and some of them will certainly find more events. A bigger increase of statistics could only come from a bigger new detector.

The IceCube Collaboration became excited when the high energy neutrino events were detected and there are now discussions of increasing the size of the detectors by a factor of more than five. This is possible if the distance between strings is significantly increased. The threshold for neutrino detection will increase (originally IceCube was designed for detection of $\mathrm{TeV}$ neutrinos) but the number of detected of high energy neutrino events will increase too. The construction of such an extension, when it happens, will take some time. The detailed design itself will take still a couple of years. The construction, although the collaboration is much better now than in the be- 
ginning, should also take several years and the new events will start coming in at higher rate after that.

There is also the development of new detection methods mostly based on the idea of Askaryan [13] about the radio emission from high energy cascades. There have already been several test experiments that have detected radio pulses of possible neutrino interactions and also from air showers. One of these experiments, ARA, is deployed at the edge of IceCube and attempts to detect high energy neutrino events in coincidence with it. The deployment of such detectors in the ice is much easier than the IceCube DOMs because they are much closer to the surface on the ice. There are also a couple of test radio detectors deployed with the Auger observatory. Radio signals detectors could replace the fluorescent detectors in the future and increase the active time of the hybrid air shower arrays by a large factor. With the development of such new techniques we hope that the effective size of all detectors will increase and this will have a positive effect on size of all event samples.

Acknowledgments I am grateful to many collegues for the long discussions of these detection. My work on atmospheric neutrinos and on the arrival directions of different types of events is funded by DOE grant DE-FG02-91ER40626.

\section{References}

[1] Eli Waxman, John N. Bahcall, Phys.Rev. D59 (1999) 023002

[2] R. Abbasi et al. (IceCube Collaboration), Phys.Rev. D83:012001 (2011)

[3] M.G. Aartsen et al. (IceCube collaboration), Phys.Rev. D91:122004 (2015)

[4] G.D. Barr et al., Phys. ReV. D70:023006 (2004)

[5] J. Learned \& S. Pakvasa, Astropart. Phys., 3, 267 (1995)

[6] www.tevcat.uchicage.ecu

[7] R. Abbasi et al. (IceCube Collaboration), Phys. Rev. D84:072001 (2011)

[8] M.G. Aartsen et al. (IceCube Collaboration), Phys. Rev. Lett., 111:021103 (2013)

[9] M.G. Aartsen et al. (IceCube Collaboration), Science, 342:1242856 (2013)

[10] M.G. Aartsen et al. (IceCube Collaboration), Phys.Rev.Lett. 113:101101 (2014)

[11] M.G. Aaertsen et al. (IceCube Collaboration), arxiv 1510.05223v2

[12] W.H. Baumgartner et al., Astrophys.J.Suppl. 207, 19 (2013)

[13] G.A. Askaryan, JETP 14:441 (1962) 


\section{DISCUSSION}

GIULIO AURIEMMA: I was wondering if you ever tried to test the statistical probability of the hypothesis that an uniform angular distribution of your neutrino events over the visible sky.

TODOR STANEV: I am not ready to answer your question with the derived probability for an excess in some regions of the sky, but the question was discussed many times by the IceCube Collaboration and the conclusion is that apart from the group of events around the Galactic center no other excess was discovered.

JONATHAN TAN: Is there any significance in spatial clustering of the source directions towards the galactic center or the galactic plane?

TODOR STANEV: The general assumption is that the sources of the high energy astrophysical neutrinos are not inside the Milky Way. On the other hand, the interactions of the galactic cosmic rays can in principle generate 1 and $2 \mathrm{PeV}$ neutrinos. I believe that the Collaboration would look more carefully at this region if there is a significant fraction of new events coming from the same area.

JIM BEALL: Will the increase of the size of the detector hurt the angular resolution of the detector ?

TODOR STANEV: No, I believe that the angular resolution will be very similar to the current one. What will change is the threshold energy for detection of astrophysical neutrinos, which will go up to about $100 \mathrm{TeV}$. 